\title{
Threshold copulas and positive dependence
}

\author{
Fabrizio Durante ${ }^{\mathrm{a}, *}$, Rachele Foschi ${ }^{\mathrm{b}, 1}$, Fabio Spizzichino ${ }^{\mathrm{b}, 1}$ \\ ${ }^{a}$ Department of Knowledge-Based Mathematical Systems, \\ Johannes Kepler University, A-4040 Linz, Austria \\ ${ }^{\mathrm{b}}$ Department of Mathematics, University "La Sapienza", I-00185 Rome, Italy
}

\begin{abstract}
Starting with a notion of positive dependence $\mathcal{P}$ and with the family of the lower threshold copulas $C_{t}$ associated with a bivariate distribution having copula $C$, we define different notions of positive dependence for $C$, reflecting the dependence properties of the copulas $C_{t}$ for some $t$.

Then, we analyze some structural aspects of lower threshold copulas and of the given definitions. Furthermore we consider several specific cases arising from relevant special choices of $\mathcal{P}$ (e.g., PQD, LTD, $\mathrm{TP}_{2}, \mathrm{PLR}$ ). Our analysis, in particular, allows us to present a number of relevant examples and counter-examples, which can be useful in the study of the tail dependence for a bivariate distribution.
\end{abstract}

Key words: Copulas, tail dependence, evolution of dependence, hyper-dependence.

\section{Introduction}

Let $(X, Y)$ be a pair of two continuous random variables whose joint distribution function is given by $F(x, y)=\mathbb{P}(X \leq x, Y \leq y)$. For every real $t$ such that $\mathbb{P}(X \leq t, Y \leq t)>0$, we consider the new distribution function

$$
F_{t}(x, y)=P(X \leq x, Y \leq y \mid X \leq t, Y \leq t)
$$

* Corresponding author.

Email addresses: fabrizio.durante@jku.at (Fabrizio Durante), foschi@mat. uniroma1. it (Rachele Foschi), fabio.spizzichino@uniroma1.it (Fabio Spizzichino).

1 The second and the third author were partially supported by Italian MIUR, in the frame of the PRIN 2006 Project "Metodi Matematici in Finanza". 
In different applied fields, in fact, interest arises in the study of the conditional distribution of $X, Y$, given the event that $X, Y$ fall under a short threshold. Below, we will also occasionally use the notation $F_{t}^{(F)}$ in order to stress that $F$ is the joint distribution function of the pair $(X, Y)$ in $(1)$.

Recently several authors studied the limit behaviour of $F_{t}$ for $t \rightarrow 0$, mainly motivated by applications to risk management: see, for example, the papers by Juri and Wüthrich $(2002,2003)$ and Charpentier and Juri (2006). In particular, the attention is focused on the properties of stochastic dependence for $F_{t}$, in the limit, also called tail-dependence properties.

In our paper, we are rather interested in the evolution of dependence properties of $F_{t}$, for $t$ spanning the interval $(0,1]$. An analysis in the same direction has been already approached by some authors (Bassan and Spizzichino, 2003; Charpentier, 2006; Foschi and Spizzichino, 2008), and, restricted to "Archimedean" models, by Pellerey (2007). Here we aim at formalizing and extending some of these results by considering, for example, different notions of positive dependence and weaker assumptions on the copula of $F$ (which, e.g., is not necessarily exchangeable), in the sense that we are going to describe.

First, we recall that a copula $C$ is the restriction to $[0,1] \times[0,1]$ of a bivariate distribution function with uniform margins. A dependence property $\mathcal{P}$ holds for a continuous joint distribution $F$ if and only if it holds for the copula associated with $F$. In other words, the copula is exactly the concept that captures the dependence of a random vector apart from its marginal behaviour (Joe, 1997; Nelsen, 2006). For this reason (see also the next Section 2), we are practically allowed, without any loss of generality, to consider, in place of $(X, Y)$, a pair of random variables $U, V$ uniformly distributed over $[0,1]$ and with joint distribution determined by $C$. We then replace $F$ with $C, C$ being a copula. Furthermore we consider, for $0<t \leq 1$, the copula $C_{t}$ defined as the copula associated with $F_{t}^{(C)}$. The copulas $C_{t}(0<t \leq 1)$ are called lower threshold copulas associated with $C$. In Section 2 we introduce some structural aspects, relevant to our analysis, concerning $C_{t}$ and the relations between $C_{t}$ and $C$.

We can now explain the purposes of our analysis.

Let $\mathcal{P}$ be a positive dependence property. The condition " $C_{t}$ satisfies $\mathcal{P}$ " (for some $t$ ) can actually be interpreted as a condition on $C$. Now, let $\Lambda$ be an interval of $(0,1]$.

Definition 1 We say that $C$ is $\langle\mathcal{P} ; \Lambda\rangle$ if $C_{t}$ is $\mathcal{P}$ for every $t \in \Lambda$. In particular, we say that $C$ is hyper- $\mathcal{P}$ if $C$ is $\langle\mathcal{P} ;(0,1]\rangle$.

An hyper- $\mathcal{P}$ property may be considered a property of positive dependence. As a main purpose of this paper we are interested in comparing the properties $\mathcal{P},\langle\mathcal{P} ; \Lambda\rangle$ and hyper- $\mathcal{P}$. 
Our study will be carried out in Section 3, where we analyze basic aspects of the Definition 1 and derive some conclusions that can be of interest in the investigation of tail dependencies. In particular we are interested in considering whether hyper- $\mathcal{P}$ coincides with some other known properties, stronger than $\mathcal{P}$. For particular choices of $\mathcal{P}$, namely PQD, LTD, $\mathrm{TP}_{2}$, PLR, we specifically analyze the properties $\langle\mathcal{P} ; \Lambda\rangle$ and hyper- $\mathcal{P}$, providing a complete comprehension of Definition 1 by means of some useful examples and counter-examples.

For a better comparison with the literature about tail dependence, in this paper we preferred to express our results in terms of distribution functions, copulas, and lower threshold copulas. In other applied fields, such as, e.g., reliability, survival analysis, interacting defaults, $X, Y$ are typically non-negative variables and, for given $\bar{F}(x, y)=\mathbb{P}(X>x, Y>y)$, one rather considers

$$
\bar{F}_{t}(x, y)=P(X>t+x, Y>t+y \mid X>t, Y>t),
$$

in place of (1). For every $t \geq 0$ such that $\mathbb{P}(X>t, Y>t)>0, \bar{F}_{t}$ is then the survival function of $(X-t, Y-t)$ conditional on the fact that $(X>t, Y>$ $t)$. Denoting by $\left(X_{t}, Y_{t}\right)$ the random pair whose survival function coincides with $\bar{F}_{t}, X_{t}$ and $Y_{t}$ are interpreted as residual lifetimes. The evolution of the dependence among $X_{t}$ and $Y_{t}$ can be studied in terms of the upper threshold copulas, that are the survival copulas of the $\bar{F}_{t}$ 's. Our results about lower threshold copulas can be equivalently reformulated for upper threshold copulas by means of a simple transformation.

\section{Threshold copulas}

Let $U, V$ be two random variables (=r.v.'s) defined on the same probability space $(\Omega, \mathcal{A}, \mathbb{P})$ and uniformly distributed on $[0,1]$, whose joint distribution function (=d.f.) is given by the copula $C$. For every $t \in(0,1]$, suppose that $C(t, t)>0$. We are interested in the conditional distribution $F_{t}$ of $(U, V)$ given that $U \leq t$ and $V \leq t$. For every $x, y \in[0, t]$, we have:

$$
F_{t}(x, y)=\mathbb{P}(U \leq x, V \leq y \mid U \leq t, V \leq t)=\frac{C(x, y)}{C(t, t)}
$$

The univariate marginal d.f.'s $G_{t}$ and $H_{t}$ are given, for every $x \in[0, t]$, by

$$
G_{t}(x)=\mathbb{P}(U \leq x, V \leq t \mid U \leq t, V \leq t)=\frac{C(x, t)}{C(t, t)}
$$

and

$$
H_{t}(x)=\mathbb{P}(U \leq t, V \leq x \mid U \leq t, V \leq t)=\frac{C(t, x)}{C(t, t)}
$$


For any fixed $t \in(0,1]$, let $h_{t}:[0,1] \rightarrow[0, t], h_{t}(u)=C(u, t)$, be the horizontal section of $C$ at the level $t$, and, analogously let $k_{t}:[0,1] \rightarrow[0, t], k_{t}(u)=$ $C(t, u)$, be the vertical section of $C$ at the level $t$. Then, we can rewrite

$$
G_{t}(x)=\frac{h_{t}(x)}{h_{t}(t)} \quad \text { and } \quad H_{t}(x)=\frac{k_{t}(x)}{k_{t}(t)}
$$

Notice that, for any fixed $t \in(0,1], h_{t}(t)=k_{t}(t)=C(t, t)$.

We now need to recall that generally, for a bivariate distribution $F(x, y)$ with marginal distributions $G(x)$ and $H(y)$, the copula associated with $F$ is the copula defined by

$$
D(u, v)=F\left(G^{[-1]}(u), H^{[-1]}(v)\right),
$$

where $G^{[-1]}$ and $H^{[-1]}$ are the pseudo-inverse functions of $G$ and $H$, respectively, also called quantile inverse (Nelsen, 2006). Then, it is obvious that

$$
F(x, y)=D(G(x), H(y)) .
$$

When $G(x)$ and $H(y)$ are continuous, $D$ is the unique copula for which Eq. (5) holds.

Now, we come back to consider the distribution $F_{t}$ defined above (by (2)) and we notice that the univariate margins $G_{t}$ and $H_{t}$ are not uniform.

The copula associated with $F_{t}$ is defined, for every $u, v \in[0,1]$, by

$$
C_{t}(u, v)=F_{t}\left(G_{t}^{[-1]}(u), H_{t}^{[-1]}(v)\right) .
$$

By recalling (3), we can obtain

$$
G_{t}^{[-1]}(u)=h_{t}^{[-1]}\left(u h_{t}(t)\right) \quad \text { and } \quad H_{t}^{[-1]}(v)=k_{t}^{[-1]}\left(v k_{t}(t)\right)
$$

where, for any fixed $t \in(0,1]$,

$$
h_{t}^{[-1]}(u)=\sup \{z \in[0,1] \mid C(z, t) \leq u\}
$$

is the pseudo-inverse of $h_{t}$, and, analogously for $k_{t}^{[-1]}$.

In view of (2) we can conclude

$$
C_{t}(u, v)=\frac{C\left(h_{t}^{[-1]}\left(u h_{t}(t)\right), k_{t}^{[-1]}\left(v k_{t}(t)\right)\right)}{C(t, t)},
$$

In the rest of the paper, we will assume that, for any fixed $t \in(0,1], h_{t}$ and $k_{t}$ are strictly increasing on $[0, t]$ and, therefore, $h_{t}^{[-1]}$ and $k_{t}^{[-1]}$ are their respective standard inverse functions on $[0, C(t, t)]$. 
Remark 2 Eq. (7) shows that, for any $t, C_{t}$ only depends on the restriction of $C$ on $[0, t]^{2}$. More generally, it is also interesting to notice that, if $C$ is the copula associated with a distribution $F$, then the copula associated with $F_{t}^{(F)}$ only depends on the restriction of $C$ on $[0, t]^{2}$.

The copulas $C_{t}$ defined by (7) are called lower threshold copula associated with $C$. In the sequel, we will denote by $\mathcal{C}$ the class of all copulas $C$ satisfying our assumptions, i.e. $C(t, t)>0$ for every $t \in(0,1]$, and $C$ has horizontal and vertical sections (at a fixed $t \in(0,1])$ strictly increasing on $[0, t]$. In particular, every $C \in \mathcal{C}$ generates a family of copulas $\left\{C_{t}\right\}_{t \in(0,1]}$, where we set $C_{1}:=C$.

Remark 3 Given a copula $C$, the left-residuum of $C$ is the function $R_{C}^{l}$ : $[0,1]^{2} \rightarrow[0,1]$ defined by $R_{C}^{l}(x, y)=\sup \{z \in[0,1] \mid C(z, x) \leq y\}$ and the right-residuum of $C$ is the function $R_{C}^{r}:[0,1]^{2} \rightarrow[0,1]$ defined by $R_{C}^{r}(x, y)=$ $\sup \{z \in[0,1] \mid C(x, z) \leq y\}$. These two functions have been proved to be useful in multivalued logic. Here, it is important to note that, by using Theorem 3.3 by Durante et al. (2007), both $R_{C}^{l}$ and $R_{C}^{r}$ are continuous in each argument with $R_{C}^{l}(t, u)=h_{t}^{[-1]}(u)$ and $R_{C}^{r}(t, u)=k_{t}^{[-1]}(u)$.

Remark 4 In previous papers, the lower and upper threshold copulas are called lower and upper tail dependence copulas. Here, we prefer to adopt a different terminology following McNeil et al. (2005, section 7.6.3), also in order to avoid confusion with the (different) notion of tail copula recently presented by Einmahl et al. (2006).

Before investigating the evolution of the dependence along the family $\left\{C_{t}\right\}_{t \in(0,1]}$, it is important to note that, with $t$ spanning $(0,1]$, the family has "no jump", in the sense that the copulas $C_{t_{0}}$ and $C_{t_{1}}$ are close each other with respect to the $L^{\infty}$-norm for sufficiently close $t_{0}$ and $t_{1}$, as stated in the following result.

Proposition 5 Let $C \in \mathcal{C}$. The mapping $\Psi:(0,1] \rightarrow \mathcal{C}, t \mapsto C_{t}$, is continuous, in the sense that, for every $(u, v) \in[0,1]^{2}, C_{t}(u, v)$ converges to $C_{t_{0}}(u, v)$ when $t$ tends to $t_{0}$.

PROOF. We have to prove that, for all $u, v \in[0,1]$ and for all $\varepsilon>0$, there exists $\delta>0$ such that, if $\left|t-t_{0}\right|<\delta$, then $\left|C_{t}(u, v)-C_{t_{0}}(u, v)\right|<\varepsilon$.

First, given $(u, v) \in[0,1]^{2}$, consider that

$$
\begin{aligned}
\left|C_{t}(u, v)-C_{t_{0}}(u, v)\right| & =\left|\frac{C(x, y)}{C(t, t)}-\frac{C\left(x_{0}, y_{0}\right)}{C\left(t_{0}, t_{0}\right)}\right| \\
& =\frac{\left|C(x, y) C\left(t_{0}, t_{0}\right)-C\left(x_{0}, y_{0}\right) C(t, t)\right|}{C(t, t) C\left(t_{0}, t_{0}\right)},
\end{aligned}
$$


where

$$
x=h_{t}^{-1}\left(u h_{t}(t)\right), y=k_{t}^{-1}\left(v k_{t}(t)\right), x_{0}=h_{t_{0}}^{-1}\left(u h_{t_{0}}\left(t_{0}\right)\right), y_{0}=k_{t_{0}}^{-1}\left(v k_{t_{0}}\left(t_{0}\right)\right),
$$

for $t, t_{0} \in(0,1],\left|t-t_{0}\right|<\delta$ for a suitable $\delta>0$. Set $\alpha:=\frac{1}{C(t, t) C\left(t_{0}, t_{0}\right)}$. We have:

$$
\begin{aligned}
& \left|C(x, y) C\left(t_{0}, t_{0}\right)-C\left(x_{0}, y_{0}\right) C(t, t)\right| \\
\leq & \left|C(x, y) C\left(t_{0}, t_{0}\right)-C(x, y) C(t, t)\right|+\left|C(x, y) C(t, t)-C\left(x_{0}, y_{0}\right) C(t, t)\right| \\
= & C(x, y)\left|C\left(t_{0}, t_{0}\right)-C(t, t)\right|+C(t, t)\left|C(x, y)-C\left(x_{0}, y_{0}\right)\right| .
\end{aligned}
$$

Since a copula is a Lipschitz function (with constant 1),

$$
\left|C\left(t_{0}, t_{0}\right)-C(t, t)\right| \leq 2\left|t-t_{0}\right|<2 \delta .
$$

Analogously,

$$
\left|C(x, y)-C\left(x_{0}, y_{0}\right)\right| \leq\left|x-x_{0}\right|+\left|y-y_{0}\right| .
$$

In order to estimate $\left|x-x_{0}\right|$ and $\left|y-y_{0}\right|$, we notice that $h_{t}^{-1}(w)$ and $k_{t}^{-1}(w)$ are alternative notations for the left- and right- residua of $C$. Then

$$
\begin{aligned}
& \left|x-x_{0}\right|=\left|R_{C}^{l}(t, u C(t, t))-R_{C}^{l}\left(t_{0}, u C\left(t_{0}, t_{0}\right)\right)\right| \\
\leq & \left|R_{C}^{l}(t, u C(t, t))-R_{C}^{l}\left(t_{0}, u C(t, t)\right)\right|+\left|R_{C}^{l}\left(t_{0}, u C(t, t)\right)-R_{C}^{l}\left(t_{0}, u C\left(t_{0}, t_{0}\right)\right)\right| .
\end{aligned}
$$

Since $R_{C}^{l}$ is continuous in each argument and

$$
u\left|C(t, t)-C\left(t_{0}, t_{0}\right)\right| \leq 2 u\left|t-t_{0}\right| \leq 2 u \delta \leq 2 \delta,
$$

it follows that we can consider a suitable $\eta$ such that

$$
\left|x-x_{0}\right| \leq \frac{\eta}{2}+\frac{\eta}{2}<\eta
$$

By the same arguments applied for $R_{C}^{r}$, we have also that $\left|y-y_{0}\right|<\eta$. Thus

$$
\left|C_{t}(u, v)-C_{t_{0}}(u, v)\right| \leq \alpha(2 \delta C(x, y)+2 \eta C(t, t)) \leq 2 \alpha(\delta+\eta) .
$$

Choosing a suitable $\eta$ such that $0<\delta<\frac{\varepsilon}{2 \alpha}-\eta$, the proof is concluded.

\section{Lower threshold copulas and generated dependence properties}

Consider $C \in \mathcal{C}$ and let $\left\{C_{t}\right\}_{t \in(0,1]}$ be the family of corresponding lower threshold copulas.

As mentioned in the Introduction, in this Section we aim at comparing, for a given dependence property $\mathcal{P}$, the properties $\mathcal{P}$, hyper $-\mathcal{P}$, and $\langle\mathcal{P} ; \Lambda\rangle$. As a first 
step, we point out some basic aspects of Definition 1. Then, for a number of relevant notions of dependence $\mathcal{P}$, we analyze the properties $\langle\mathcal{P} ; \Lambda\rangle$, hyper- $\mathcal{P}$, and relations among them.

Remark 6 Let $\Lambda$ be an arbitrary proper subset of $(0,1], \bar{\Lambda}$ being the closure of $\Lambda$, and consider the two conditions $\langle\mathcal{P} ; \Lambda\rangle,\langle\mathcal{P} ; \bar{\Lambda}\rangle$. As an immediate consequence of the continuity property in Proposition 5, these two conditions coincide. We may then argue that, in considering the property $\langle\mathcal{P} ; \Lambda\rangle$, we can limit attention to the cases when $\Lambda$ is a closed subset of $(0,1]$, i.e., an closed interval or a union of disjoint closed intervals.

Actually we are only interested in subsets $\Lambda$ of the form $(0, \lambda]$, for some constant $\lambda \in(0,1]$.

We notice that the condition $\langle\mathcal{P} ;(0, \lambda]\rangle$ for a copula $C$ has the following immediate meaning: it means that $C$ may not satisfy $\mathcal{P}$ and that the property $\mathcal{P}$ possibly holds for all $C_{t}$, with $t$ below a given $\lambda$. This notion can be of interest in the field of tail dependence. In fact we are typically interested in proving that $C_{t}$ satisfies a dependence property $\mathcal{P}$ in the limit for $t \rightarrow 0$. Thus, proving that $C$ is $\langle\mathcal{P} ;(0, \lambda]\rangle$ guarantees the above condition without explicit computation of $\lim _{t \rightarrow 0} C_{t}$.

As a consequence of Eq. (7) (see Remark 2), we can state that $\langle\mathcal{P} ; \Lambda\rangle$ only depends on the behaviour of $C$ on $\Lambda^{2}$ (see, in this respect, Propositions 9, 13, 14,18 below).

Generally, the property $\langle\mathcal{P} ; \Lambda\rangle$ does not imply $\mathcal{P}$ (see Example 12) nor does $\mathcal{P}$ imply $\langle\mathcal{P} ; \Lambda\rangle$ (see Example 10).

As far as the property hyper- $\mathcal{P}$ is concerned, we notice that hyper- $\mathcal{P} \Rightarrow \mathcal{P}$, just by definition. We can also state a sufficient (not a necessary) condition on $\mathcal{P}$ under which the implication $\mathcal{P} \Rightarrow$ hyper- $\mathcal{P}$ even holds. Let $\mathcal{P}$ and $\mathcal{P}^{\prime}$ be two different properties of positive dependence. We have the following result.

Proposition 7 If $\mathcal{P}$ is equivalent to hyper- $\mathcal{P}^{\prime}$, then $\mathcal{P}$ is equivalent to hyper- $\mathcal{P}$

PROOF. For a dependence property $\mathcal{Q}$, it can be shown that $C$ hyper- $\mathcal{Q}$ implies $C_{t}$ hyper- $\mathcal{Q}$ for any $t$. We can then write the following chain of implications:

$$
\begin{aligned}
C \text { satisfies } \mathcal{P} & \Rightarrow C \text { is hyper }-\mathcal{P}^{\prime} \Rightarrow C_{t} \text { is hyper }-\mathcal{P}^{\prime} \\
& \Leftrightarrow \forall t \quad C_{t} \text { is } \mathcal{P} \Leftrightarrow C \text { is hyper }-\mathcal{P},
\end{aligned}
$$

which proves the desired assertion. 
Two different examples of this last situation are provided by the properties of $\mathrm{TP}_{2}$ and PLR (as we will see below).

Now, we study the properties $\langle\mathcal{P} ; \Lambda\rangle$ and hyper-P for relevant notions of dependence recalled below (see also (Kimeldorf and Sampson, 1989; Nelsen, 2006) for a more complete overview).

Definition 8 Let $C \in \mathcal{C}$ the d.f. of the random pair $(U, V)$.

- $C$ is PQD (i.e. positively quadrant dependent) if, and only if, $C(u, v) \geq u v$ for every $(u, v) \in[0,1]^{2}$.

- $C$ is $\operatorname{LTD}(V \mid U)$ (i.e. $V$ is left tail decreasing in $U$ ) if, and only if, $u \mapsto$ $\frac{C(u, v)}{u}$ is decreasing on $[0,1]$. Analogously, $C$ is $\operatorname{LTD}(U \mid V)$ (i.e. $U$ is left tail decreasing in $V)$ if, and only if, $v \mapsto \frac{C(u, v)}{v}$ is decreasing on $[0,1]$.

- $C$ is $\mathrm{TP}_{2}$ (i.e. totally positive of order 2) if, and only if, for all $x, x^{\prime}, y, y^{\prime}$ in $[0,1], x \leq x^{\prime}$ and $y \leq y^{\prime}$

$$
C(x, y) C\left(x^{\prime}, y^{\prime}\right) \geq C\left(x, y^{\prime}\right) C\left(x^{\prime}, y\right) .
$$

- $C$ is PLR (i.e. positively likelihood ratio dependent) if, and only if, $C$ is absolutely continuous and its density $c$ satisfies (8), with $C$ replaced by $c$.

The following chain of implications holds (and none of the converse implications is satisfied)

$$
\mathrm{PLR} \quad \Rightarrow \quad \mathrm{TP}_{2} \quad \Rightarrow \quad \operatorname{LTD}(U \mid V)(\operatorname{or} \operatorname{LTD}(V \mid U)) \quad \Rightarrow \quad \mathrm{PQD} \text {. }
$$

Let $\Lambda=(0, \lambda]$ be an interval of $(0,1]$ and consider $C \in \mathcal{C}$.

Proposition $9 C$ is $\langle\mathrm{PQD} ; \Lambda\rangle$ if, and only if, $C$ satisfies

$$
C(x, y) C(t, t) \geq C(x, t) C(t, y)
$$

for every $x, y, t \in \Lambda, x \leq t$ and $y \leq t$.

PROOF. By definition, $C$ is $\langle\mathrm{PQD} ; \Lambda\rangle$ means that $C_{t}(u, v) \geq u v$ for all $t \leq \lambda$, that is $C\left(h_{t}^{-1}\left(u h_{t}(t)\right), k_{t}^{-1}\left(v k_{t}(t)\right)\right) \geq u v C(t, t)$. Putting $x=h_{t}^{-1}\left(u h_{t}(t)\right), y=$ $k_{t}^{-1}\left(v k_{t}(t)\right)$, we get

that is inequality (9).

$$
C(x, y) \geq \frac{h_{t}(x)}{h_{t}(t)} \frac{k_{t}(y)}{k_{t}(t)} h_{t}(t)
$$

According to the general Definition 1, the copulas $\Pi(u, v)=u v$ and $M(u, v)=$ $\min (u, v)$ are hyper-PQD. 
It is also easy to show that, if $C$ is $\mathrm{TP}_{2}$, then it is hyper-PQD. Moreover, as stated in general before, if $C$ is hyper-PQD, then it is PQD. The converse implication is false, as the following example shows.

Example 10 Let $C$ be the copula given by $C(u, v)=\min \left(u, v, \frac{u^{2}+v^{2}}{2}\right)$. Then $C$ is $\mathrm{PQD}$, but not $\langle\mathrm{PQD} ; \Lambda\rangle$ for $\lambda=\frac{3}{5}$. In fact, by considering $x=y=\frac{1}{2}$, we obtain that

$$
C(x, y) C(t, t)=\frac{36}{100} \frac{25}{100}<\left(\frac{61}{200}\right)^{2}=C(x, t) C(t, y) .
$$

Therefore, $C(u, v) \geq u v$ on $[0,1]^{2}$, but $C_{\frac{3}{5}}(u, v)<u v$ for some $(u, v) \in[0,1]^{2}$. Actually, in view of Proposition 5, for every $t$ belonging to a neighbourhood of $\frac{3}{5}, C_{t}$ is not PQD.

It is interesting to note that, for an Archimedean copula $C(u, v)=\varphi^{-1}(\varphi(u)+$ $\varphi(v))$ Nelsen (2006), the notions of $\mathrm{TP}_{2}$ and hyper-PQD coincide. Furthermore, these conditions are equivalent to $t \mapsto \varphi\left(e^{-t}\right)$ being convex: the proof can be derived directly from Alsina et al. (2006, section 4.5).

We recall that the upper and lower bounds of a PQD copula are respectively $M(u, v)=\min (u, v)$ and $\Pi(u, v)=u v$. Since, in general, a PQD copula is not hyper-PQD, the lower bound for $C$ is not preserved for $C_{t}$. But, also in this case, we can obtain for $C$ a lower bound better than $W(u, v)=\max (u+v-$ $1,0)$.

Proposition 11 If $C$ is $\mathrm{PQD}$, then, for every $t \in(0,1]$, we have that

$$
C_{t}(u, v) \geq \max \left(u v t^{3}, \max (u+v-1,0)\right) .
$$

PROOF. Since $C$ is $\mathrm{PQD}, u v \leq C(u, v) \leq \min (u, v)$. In particular, $u t \leq$ $h_{t}(u) \leq \min (u, t)$ and, consequently,

$$
u \leq h_{t}^{-1}(u) \leq \min \left(\frac{u}{t}, t\right) \quad \text { for all } u \in[0, t] .
$$

Since the fact that an analogous inequality can be proved for $k_{t}$, we obtain

$$
C_{t}(u, v) \geq \frac{C\left(h_{t}^{-1}\left(u t^{2}\right), k_{t}^{-1}\left(v t^{2}\right)\right)}{t} \geq \frac{h_{t}^{-1}\left(u t^{2}\right) \cdot k_{t}^{-1}\left(v t^{2}\right)}{t} \geq u v t^{3}
$$

and hence (10) holds.

Now, note that if $C$ satisfies $\langle\mathrm{PQD},(0, \lambda]\rangle$ for a given $\lambda<1$, then $C$ needs not be PQD. 
Example 12 Consider, for example, the copula $C$ given by

$$
C(u, v)= \begin{cases}\frac{u v}{\lambda}, & (u, v) \in[0, \lambda]^{2}, \\ \lambda+(1-\lambda) C^{\prime}\left(\frac{u-\lambda}{1-\lambda}, \frac{v-\lambda}{1-\lambda}\right), & (u, v) \in[\lambda, 1]^{2}, \\ \min (u, v), & \text { otherwise, }\end{cases}
$$

where $C^{\prime}(u, v)=u v[1-(1-u)(1-v)]$ is a copula that is not $\mathrm{PQD}$, and hence nor $C$ is PQD. Actually, $C$ is an ordinal sum of the copulas $\Pi$ and $C^{\prime}$ with respect to the partition $([0, \lambda],[\lambda, 1])$. Now, compute $C_{t}(u, v)$ for any $t \in(0, \lambda]$. We obtain $C_{t}(u, v)=\frac{\lambda}{t^{2}} \cdot C(u t, v t)=u v$. It follows that $C_{t}$ is PQD for any $t \leq \lambda$, even if $C$ is not $\mathrm{PQD}$.

Now, let us consider the other dependence properties, LTD, $\mathrm{TP}_{2}, \mathrm{PLR}$. Similarly to Proposition 9, the following result holds for the LTD property.

Proposition $13 C$ is $\langle\operatorname{LTD}(V \mid U) ; \Lambda\rangle$ if, and only if,

$$
C(x, y) C\left(x^{\prime}, t\right) \geq C(x, t) C\left(x^{\prime}, y\right)
$$

for all $t \in \Lambda, x, x^{\prime}, y \in[0, t]$ such that $x \leq x^{\prime}$.

An analogous condition holds for $\langle\operatorname{LTD}(U \mid V) ; \Lambda\rangle$.

PROOF. Since $C$ is $\langle\operatorname{LTD}(V \mid U) ; \Lambda\rangle$, then, for any $u \leq u^{\prime}$,

$$
\frac{C\left(h_{t}^{-1}(u C(t, t)), k_{t}^{-1}(v C(t, t))\right)}{u} \geq \frac{C\left(h_{t}^{-1}\left(u^{\prime} C(t, t)\right), k_{t}^{-1}(v C(t, t))\right)}{u^{\prime}},
$$

that is

$$
\frac{C(t, t)}{C(x, t)} C(x, y) \geq C\left(x^{\prime}, y\right) \frac{C(t, t)}{C\left(x^{\prime}, t\right)}
$$

for all $x, x^{\prime}, y \leq t$ such that $x \leq x^{\prime}$. Thus, we obtain (11). Part (b) can be proved in the same way.

Examples of hyper-LTD $(V \mid U)$ copulas are $\Pi(u, v)=u v$ and $M(u, v)=$ $\min (u, v)$. Since they are symmetric with respect to $u$ and $v$, they also are hyper-LTD $(U \mid V)$.

We now come to the $\mathrm{TP}_{2}$. For such a property, we find some differences from the other dependence properties taken in account until now. As we can easily deduce from the following proposition, $\left\langle\mathrm{TP}_{2} ; \Lambda\right\rangle$ is a weaker property than $\mathrm{TP}_{2}$. We can build a copula satisfying $\left\langle\mathrm{TP}_{2} ; \Lambda\right\rangle$, but not $\mathrm{TP}_{2}$, following Example 12. 
Proposition $14 A$ copula $C$ is $\left\langle\mathrm{TP}_{2} ; \Lambda\right\rangle$ if, and only if, $C$ satisfies (8) on $\Lambda^{2}$.

PROOF. By definition, $C$ is $\left\langle\mathrm{TP}_{2} ; \Lambda\right\rangle$ if, and only if, for every $t \in \Lambda C_{t}$ is $\mathrm{TP}_{2}$, i.e.

$$
C_{t}(u, v) C_{t}\left(u^{\prime}, v^{\prime}\right) \geq C_{t}\left(u, v^{\prime}\right) C_{t}\left(u^{\prime}, v\right),
$$

for all $u \leq u^{\prime}$ and $v \leq v^{\prime}$. Writing explicitly the multiplicands and making suitable substitutions, we obtain that, for every $x, x^{\prime}, y, y^{\prime} \in[0, t], x \leq x^{\prime}$ and $y \leq y^{\prime}$

$$
C(x, y) C\left(x^{\prime}, y^{\prime}\right) \geq C\left(x, y^{\prime}\right) C\left(x^{\prime}, y\right),
$$

which is the desired assertion.

In particular, $C$ is hyper- $\mathrm{TP}_{2}$ if, and only if, $C$ is $\mathrm{TP}_{2}$, as stated by the following corollary.

Corollary 15 Let $C \in \mathcal{C}$ be the d.f. of the random pair $(U, V)$. Then the following statements are equivalent:

(a) $C$ is $\mathrm{TP}_{2}$;

(b) $C$ is hyper $-\mathrm{TP}_{2}$;

(c) $C$ is hyper $-\operatorname{LTD}(V \mid U)$ and hyper- $\operatorname{LTD}(U \mid V)$.

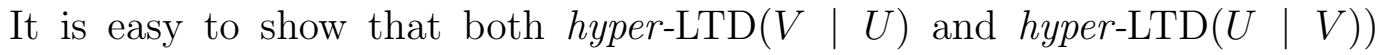
separately imply that $C$ is PQD.

We note that, in order to obtain for $\mathrm{C}$ the stronger property $\mathrm{TP}_{2}$, both hyper$\operatorname{LTD}(V \mid U)$ and hyper-LTD $(U \mid V)$ have to be satisfied. Statement (c) can be simplified if we have a condition that guarantees

$$
\operatorname{LTD}(V \mid U) \Leftrightarrow \operatorname{LTD}(U \mid V)
$$

(and therefore hyper $-\operatorname{LTD}(V \mid U) \Leftrightarrow$ hyper $-\operatorname{LTD}(U \mid V)$ ). In this case we simply say that $C$ is LTD (hyper-LTD). A sufficient condition for (12) is $C$ being exchangeable.

Corollary 16 Let $C \in \mathcal{C}$ be the d.f. of the exchangeable random pair $(U, V)$. Then the following statements are equivalent:
(a) $C$ is $\langle\operatorname{LTD}(V \mid U) ; \Lambda\rangle$;
(b) $C$ is $\langle\operatorname{LTD}(U \mid V) ; \Lambda\rangle$;
(c) $C$ satisfies (8) on $\Lambda^{2}$.

In particular, $C$ hyper $-\operatorname{LTD}(V \mid U)$ (resp. hyper-LTD $(U \mid V)$ ) is equivalent to $C$ being $\mathrm{TP}_{2}$. 
Finally, we consider the PLR property, which can be introduced only for a copula $C$ that is absolutely continuous on $[0,1]^{2}$, viz.

$$
C(u, v)=\int_{0}^{u} \int_{0}^{v} \partial_{12}^{2} C(\gamma, \theta) \mathrm{d} \gamma \mathrm{d} \theta
$$

where $\partial_{12}^{2} C$ denotes the second mixed derivative of $C$.

In order to state some properties about the family $\left\{C_{t}\right\}_{t \in(0,1]}$, it is hence important to consider whether the absolute continuity is preserved by every $C_{t}$.

Proposition 17 Let $C$ be a copula having non-zero first derivatives almost everywhere on $[0,1]^{2}$. If $C$ is absolutely continuous, then $C_{t}$ is absolutely continuous for every $t \in(0,1]$.

PROOF. For every $t \in(0,1]$, the second mixed derivative of $C_{t}$ is given by:

$$
\partial_{12}^{2} C_{t}(u, v)=\frac{C(t, t) \partial_{12}^{2} C\left(h_{t}^{-1}\left(u h_{t}(t)\right), k_{t}^{-1}\left(v k_{t}(t)\right)\right)}{\partial_{1} C\left(h_{t}^{-1}\left(u h_{t}(t)\right), t\right) \cdot \partial_{2} C\left(t, k_{t}^{-1}\left(v k_{t}(t)\right)\right)} .
$$

We have to check the equality

$$
C_{t}(u, v)=\int_{0}^{u} \int_{0}^{v} \partial_{12}^{2} C_{t}(\gamma, \theta) \mathrm{d} \gamma \mathrm{d} \theta
$$

Applying (7) and (14), we obtain

$$
\frac{C(x, y)}{C(t, t)}=\int_{0}^{u} \int_{0}^{v} \frac{C(t, t) \partial_{12}^{2} C\left(h_{t}^{-1}\left(\gamma h_{t}(t)\right), k_{t}^{-1}\left(\theta k_{t}(t)\right)\right)}{\partial_{1} C\left(h_{t}^{-1}\left(\gamma h_{t}(t)\right), t\right) \partial_{2} C\left(t, k_{t}^{-1}\left(\theta k_{t}(t)\right)\right)} \mathrm{d} \gamma \mathrm{d} \theta .
$$

Changing variables by means of

$$
\xi=h_{t}^{-1}\left(\gamma h_{t}(t)\right), \quad \eta=k_{t}^{-1}\left(\theta k_{t}(t)\right),
$$

we obtain

which is the desired assertion.

$$
C(x, y)=\int_{0}^{x} \int_{0}^{y} \partial_{12}^{2} C(\xi, \eta) \mathrm{d} \xi \mathrm{d} \eta
$$

Proposition $18 C$ is $\langle\mathrm{PLR} ; \Lambda\rangle$ if, and only if, $\partial_{12}^{2} C$ satisfies (8) on $\Lambda^{2}$.

PROOF. By definition, $C$ is $\langle\mathrm{PLR} ; \Lambda\rangle$ if, and only if, for every $t \in \Lambda, C_{t}$ is PLR, i.e.

$$
\frac{\partial^{2} C_{t}(u, v)}{\partial u \partial v} \frac{\partial^{2} C\left(u^{\prime}, v^{\prime}\right)}{\partial u \partial v} \geq \frac{\partial^{2} C\left(u^{\prime}, v\right)}{\partial u \partial v} \frac{\partial^{2} C\left(u, v^{\prime}\right)}{\partial u \partial v}
$$

for every $u, u^{\prime}, v, v^{\prime} \in[0,1], u \leq u^{\prime}$ and $v \leq v^{\prime}$. Using Eq. (13), after some simplification, we obtain the desired assertion. 
Analogously to $\mathrm{TP}_{2}, C$ PLR is equivalent to $C$ hyper-PLR. Moreover, there exists a copula $C$ such that $C$ is not PLR, even if $C$ is $\langle$ PLR; $(0, \lambda]\rangle$ for $\lambda<1$.

The relationships among dependence properties and related hyper-dependence properties are summarized here.

\begin{tabular}{|c|c|c|c|c|c|c|c|}
\hline$\langle\mathrm{PLR} ; \Lambda\rangle$ & $\Rightarrow$ & $\left\langle\mathrm{TP}_{2} ; \Lambda\right\rangle$ & $\Rightarrow$ & $\langle\operatorname{LTD}(V \mid U) ; \Lambda\rangle$ & $($ or $\langle\operatorname{LTD}(V \mid U) ; \Lambda\rangle)$ & $\Rightarrow$ & $\langle\mathrm{PQD} ; \Lambda\rangle$ \\
\hline$\Uparrow$ & & $\Uparrow$ & & & 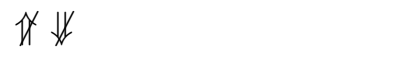 & & $\not \Uparrow \Downarrow$ \\
\hline PLR & $\Rightarrow$ & $\mathrm{TP}_{2}$ & $\Rightarrow$ & $\operatorname{LTD}(U \mid V)$ & $($ or $\operatorname{LTD}(V \mid U))$ & $\Rightarrow$ & PQD \\
\hline$\Uparrow$ & & $\Uparrow$ & & & $\Uparrow$ & & $\Uparrow$ \\
\hline \multirow[t]{3}{*}{ hyper-PLR } & $\Rightarrow$ & hyper- $\mathrm{TP}_{2}$ & $\Rightarrow$ & hyper-LTD $(U \mid V)$ & (or hyper-LTD $(V \mid U)$ ) & $\Rightarrow$ & hyper-PQD \\
\hline & & & & & $\Downarrow$ & & $\Downarrow$ \\
\hline & & & & $\langle\operatorname{LTD}(V \mid U) ; \Lambda\rangle$ & $($ or $\langle\operatorname{LTD}(V \mid U) ; \Lambda\rangle)$ & $\Rightarrow$ & $\langle\mathrm{PQD} ; \Lambda\rangle$ \\
\hline
\end{tabular}

\section{References}

Alsina, C., Frank, M. J., Schweizer, B., 2006. Associative Functions: Triangular Norms and Copulas. World Scientific, Singapore.

Bassan, B., Spizzichino, F., 2003. On some properties of dependence and aging for residual lifetimes in the exchangeable case. In: Mathematical and statistical methods in reliability (Trondheim, 2002). Vol. 7 of Ser. Qual. Reliab. Eng. Stat. World Sci. Publ., River Edge, NJ, pp. 235-249.

Charpentier, A., 2006. Dependence structure and limiting results: some applications in finance and insurance. Ph.D. thesis, Katholieke Universiteit Leuven.

Charpentier, A., Juri, A., 2006. Limiting dependence structures for tail events, with applications to credit derivatives. J. Appl. Probab. 43 (2), 563-586.

Durante, F., Klement, E., Mesiar, R., Sempi, C., 2007. Conjunctors and their residual implicators: characterizations and construction methods. Mediterr. J. Math 4 (3), 343-356.

Einmahl, J., de Haan, L., Li, D., 2006. Weighted approximations of tail copula processes with application to testing the bivariate extreme value condition. Ann. Statist. 34 (4), 1987-2014.

Foschi, R., Spizzichino, F., 2008. Semigroups of semicopulas and evolution of dependence at increase of age. Mathware \& Soft Computing, under revision.

Joe, H., 1997. Multivariate models and dependence concepts. Vol. 73 of Monographs on Statistics and Applied Probability. Chapman \& Hall, London.

Juri, A., Wüthrich, M., 2003. Tail dependence from a distributional point of view. Extremes 6 (3), 213-246 (2004).

Juri, A., Wüthrich, M. V., 2002. Copula convergence theorems for tail events. Insurance Math. Econom. 30, 405-420. 
Kimeldorf, G., Sampson, A. R., 1989. A framework for positive dependence. Ann. Inst. Statist. Math. 41 (1), 31-45.

McNeil, A., Frey, R., Embrechts, P., 2005. Quantitative risk management. Concepts, techniques and tools. Princeton Series in Finance. Princeton University Press, Princeton, NJ.

Nelsen, R., 2006. An introduction to copulas, 2nd Edition. Springer Series in Statistics. Springer, New York.

Pellerey, F., 2007. On aging properties for bivariate lifetimes with Archimedean survival copulas. Tech. Rep. 21, Politecnico di Torino. 